CORRIGENDUM

doi:10.1038/nature11864

\title{
Corrigendum: The deubiquitinase USP9X suppresses pancreatic ductal adenocarcinoma
}

Pedro A. Pérez-Mancera, Alistair G. Rust, Louise van der Weyden, Glen Kristiansen, Allen Li, Aaron L. Sarver, Kevin A. T. Silverstein, Robert Grützmann, Daniela Aust, Petra Rümmele, Thomas Knösel, Colin Herd, Derek L. Stemple, Ross Kettleborough, Jacqueline A. Brosnan, Ang Li, Richard Morgan, Spencer Knight, Jun Yu, Shane Stegeman, Lara S. Collier, Jelle J. ten Hoeve, Jeroen de Ridder, Alison P. Klein, Michael Goggins, Ralph H. Hruban, David K. Chang, Andrew V. Biankin, Sean M. Grimmond, Australian Pancreatic Cancer Genome Initiative, Lodewyk F. A. Wessels, Stephen A. Wood, Christine A. Iacobuzio-Donahue, Christian Pilarsky, David A. Largaespada, David J. Adams \& David A. Tuveson

Nature 486, 266-270 (2012); doi:10.1038/nature11114

In this Letter, several authors from the Australian Pancreatic Cancer Genome Initiative were inadvertently omitted: Christopher J. Scarlett and Warren Kaplan from the The Kinghorn Cancer Centre; and Aldo Scarpa from the ARC-NET Center for Applied Research on Cancer. Amitabha Das was incorrectly listed as Amithabad Das. Furthermore, the correct address for the ARC-NET Center for Applied Research on Cancer, University of Verona, is: Policlinico GB Rossi, Piazzale LA Scuro 10, 37134 Verona, Italy. In the acknowledgements section, S.A.W. was supported by the National Health and Medical Research Council of Australia (NHMRC); and the APGI investigators by the University of Verona and Italian Ministry of University and Research (FIRB RBAP10AHJB). These errors have been corrected in the HTML and PDF of the original paper. 